\section{Ventilation inhomogeneities assessed by the multibreath washout (MBW) technique}

\author{
Philipp Latzin, ${ }^{1}$ Cindy Thamrin, ${ }^{1}$ Richard Kraemer ${ }^{2}$
}

Intrapulmonary gas distribution and mixing is an important functional property of the lungs and can be characterised by the multibreath washout (MBW) technique. By computing indices from the entire washout curve, ventilation inhomogeneities can be characterised. ${ }^{1-5}$ Developed in the late 1980s for use in children, ${ }^{6-8} \mathrm{MBW}$ measurements are taken during tidal breathing and have been used in infants ${ }^{9-12}$ as well as preschool children. ${ }^{712}$ This technique is especially advantageous in the latter as only a few tests exist for measuring pulmonary function without active subject cooperation.

The MBW allows us to measure functional residual capacity ( FRC $\left._{M B W}\right)$, which can be used in combination with the plethysmographic intrathoracic gas volume $\left(\right.$ FRC $\left._{\text {pleth }}\right)$ to determine the amount of trapped gas in the lungs $\left(\mathrm{V}_{\mathrm{TG}}\right){ }^{14-17} \mathrm{In}$ addition, using the washout curve, different measures of ventilation inhomogeneity can be obtained. The lung clearance index (LCI) is defined as the number of lung volume turnovers (cumulative expired volume divided by FRC). ${ }^{235}$ Other more sophisticated indices include the moment ratios $\left(\mathrm{m}_{1}: \mathrm{m}_{0}, \mathrm{~m}_{2}: \mathrm{m}_{0}\right),{ }^{4-8} 101819$ mixing ratio (ratio between the actual and ideal breaths needed to lower the tracer gas to a defined end-tidal concentration in relation to the starting concentration $)^{1320}$ and the alveolar-based gas dilation number. ${ }^{19} 21$

At present, LCI is the most commonly used of these indices, partly because it is arguably the most intuitive and easiest to be communicated to patients. Of note, these indices of intrapulmonary gas mixing are independent of age. Moreover, LCI has been shown in patients with cystic fibrosis (CF) to be more sensitive than spirometry for detecting changes in small airways, ${ }^{13}$ to be the earliest indicator of disease progression during longitudinal follow-up, ${ }^{16}$ and is ideal for detecting

\footnotetext{
${ }^{1}$ Division of Paediatric Respiratory Medicine, Department of Paediatrics, University of Berne, Inselspital, Berne, Switzerland; ${ }^{2}$ Head of the Department of Paediatrics, University of Berne, Inselspital, Berne, Switzerland

Correspondence to: Professor R Kraemer, Department of Paediatrics, University of Berne, Inselspital, 3010 Berne, Switzerland; richard.kraemer@insel.ch
}

changes early in life, monitoring the effect of treatment and tracking lung disease with age. ${ }^{1012161822} 23$

The development of computers and advances in the knowledge of physiopathology have accelerated the technical evolution of the MBW technique, including derived methods such as the analysis of slope III which yields even more detailed information regarding inhomogeneity. 22245 The equipment used to date, however, has constraints that have prevented a more widespread application of MBW. The so-called nitrogen washout requires breathing of $100 \%$ oxygen to wash out the resident nitrogen in the lungs which can change the breathing pattern and influence the results. ${ }^{26}$ The use of inert gases such as helium or sulfur hexafluoride $\left(\mathrm{SF}_{6}\right)$ (ie, those not absorbed by the pulmonary blood stream) overcomes this problem but requires a complete wash-in of the respective gas. This leads to a long measurement duration, especially in disease, and a possible source of error arises as an incomplete wash-in would result in subsequent underestimation of the true FRC, again particularly in diseased subjects. ${ }^{8}$ Moreover, the mass spectrometer (MS) is currently the conventional gold standard used to assess the concentration of these inert gases, which is relatively costly, bulky, needs to operate under particular physical conditions and requires customised set-up of the technical equipment and the software as no commercial system is currently available. Besides nitrogen and infrared analysers, another commercially available equipment for MBW uses a validated ultrasonic flowmeter (USFM) which simultaneously determines the density of the passing gas mixture ${ }^{27}$ and calculates the corresponding concentration of the known constituent gases from this. However, this equipment has mainly been used in infants, and only recently has a study in older children been published comparing the USFM system with the MS in a side-stream sample. ${ }^{11}$

In this issue of Thorax, Holsley and coworkers $^{28}$ report a new measurement device with potential advantages over existing measurement equipment (see page 135). The Innocor device was originally used to measure cardiac output, and makes use of differences in infrared absorption spectra to measure gas concentrations. Unlike previous $\mathrm{SF}_{6}$ infrared analysers used for $\mathrm{MBW}^{29}{ }^{30}$ it measures carbon dioxide simultaneously in addition to $\mathrm{SF}_{6}$. The set-up is quite similar to the side-stream USFM, where the sampling is done via a Nafion tube in order to eliminate the effect of temperature and humidity, and is considerably less bulky than the MS. It has a high signal-to-noise ratio, good resolution and a low drift that is superior to that of the MS and possibly equivalent to that of the USFM. It also detects $\mathrm{SF}_{6}$ at one-twentieth of the gas concentration operating range of the MS and USFM, which means that only a small amount of gas is required for measurements. This has benefits both from an economic and environmental point of view: $\mathrm{SF}_{6}$ is an expensive gas and its effect on the ozone layer has been raised as an issue in recent times. ${ }^{31}$ While this is a negligible problem in infants, it becomes of greater significance given the larger amount of gas needed for use in older children. The device is already commercially available, although so far only for exercise testing, ${ }^{32}$ and thus adaptations to the set-up as well as analysis software are needed for use in the MBW technique. Although there was a tenuous dependency with age, Horsley et al were able to show good acceptability, reproducibility, and sensitivity in detecting CF with this device in adults. ${ }^{28}$

On the other hand, there are apparent drawbacks to the device. In its present implementation the Innocor device cannot measure oxygen or helium. In addition, the apparatus dead space is higher than that currently proposed by the ATS/ ERS standards for preschool measurements $^{33}$ (and even more so in infants), and limits its use in very sick patients with $\mathrm{CF}$ with $\mathrm{CO}_{2}$ retention. The fact that a dead space/tidal volume ratio of $>0.2^{34}$ may artificially increase indices of ventilation inhomogeneities is important to consider when using this equipment in patients with small tidal volumes, such as those with neuromuscular disorders. Neither the relatively high dead space nor the slow response time fulfil measurement specifications required for use in infants as well as preschool children. ${ }^{35}$ In this age group, the USFM may still be the better choice than the two pneumotachograph-based systems in terms of flow range and resolution, as well as the need 
for correction of the delay between flow and gas analyser signal. ${ }^{36}{ }^{37}$ However, the Innocor device may prove to be promising for the measurement of gas concentration and for use in older children and adults.

Before this or any other system can be used more widely, some issues still need to be resolved regarding measurement and analysis. Standards for performing MBW measurements exist for infants (only for the use of nitrogen) ${ }^{38}$ and for preschool children, ${ }^{33}$ but these do not take into account differences between equipment. Moreover, no international reference values for MBW parameters currently exist. There is therefore an urgent need for standardisation of MBW measurements and availability of reference values in older subjects as well. It has been known for a long time that indices of lung ventilation depend on the ratio of ventilated dead space and tidal volume, ${ }^{834}$ an interrelationship which is even more important in small infants with low tidal volumes, possibly explaining higher observed LCI values in younger infants. ${ }^{12}$ This is important to consider when measuring with or without a filter, and with a mouthpiece or facemask. The influence of the latter option and of different tracer gases on the results is unknown. Here again, a standardised index independent of breathing pattern and dead space/tidal volume ratio would be preferable, ${ }^{19}{ }^{21}$ especially since it is unclear which of the proposed indices are superior in discriminating between health and disease. ${ }^{8}$ Other points that may impact on the results are the suctioning of a constant flow for the purposes of gas sampling between the flow sensor and the patient-as used in the side-stream set-up and the MS-and the different effects of temperature and humidity as well as corrections for BTPS conditions. The criteria for determining the end of washout is currently arbitrarily defined, dating from the days of nitrogen washouts. ${ }^{3}{ }^{4}$ Finally, the long duration of the test does not yet allow its widespread use in daily clinical practice.

A simple and non-invasive method like the MBW technique holds much promise. Measurements of intrapulmonary inhomogeneity have good repeatability and acceptability. Clinically, it might also be useful in patient groups other than CF and for monitoring environmental effects on small airway function, as shown for tobacco smoke. ${ }^{39}$ Although there are still many issues to be resolved, the introduction and careful validation of the Innocor device as reported by Horsley et $a^{28}$ is an important step towards acceleration of the renaissance of the MBW technique. This report hopefully will stimulate researchers and manufacturers to improve current measurement equipment and analysis software in order to help standardisation and allow comparison between centres.

Funding: None.

Competing interests: None.

Thorax 2008;63:98-99. doi:10.1136/thx.2007.085332

\section{REFERENCES}

1. Fowler WS, Cornish ER Jr, Kety SS. Lung function studies. VIII. Analysis of alveolar ventilation by pulmonary $\mathrm{N}_{2}$ clearance curves. J Clin Invest 1952;31:40-50.

2. Becklake MR. A new index of the intrapulmonary mixture of inspired air. Thorax 1952;7:111-6.

3. Bouhuys A. Pulmonary nitrogen clearance in relation to age in healthy males. J App/ Physiol 1963;18:297-300.

4. Saidel GM, Salmon RB, Chester EH. Moment analysis of multibreath lung washout. J Appl Physiol 1975; 38:328-34.

5. Fleming GM, Chester EH, Saniie J, et al. Ventilation inhomogeneity using multibreath nitrogen washout: comparison of moment ratios and other indexes. $\mathrm{Am}$ Rev Respir Dis 1980;121:789-94.

6. Hutchison AA, Sum AC, Demis TA, et al. Moment analysis of multiple breath nitrogen washout in children. Am Rev Respir Dis 1982;125:28-32.

7. Wall MA. Moment analysis of multibreath nitrogen washout in young children. J Appl Physiol 1985:59:274-9.

8. Kraemer R, Meister B. Fast real-time moment-ratio analysis of multibreath nitrogen washout in children. J Appl Physiol 1985:59:1137-44.

9. Shao H, Sandberg K, Sjoqvist BA, et al. Moment analysis of multibreath nitrogen washout in healthy preterm infants. Pediatr Pulmonol 1998;25:52-8.

10. Aurora P, Kozlowska W, Stocks J. Gas mixing efficiency from birth to adulthood measured by multiple-breath washout. Respir Physiol Neurobiol 2005;148:125-39.

11. Fuchs SI, Buess C, Lum S, et al. Multiple breath washout with a sidestream ultrasonic flow sensor and mass spectrometry: a comparative study. Pediatr Pulmonol 2006;41:1218-25.

12. Lum S, Gustafsson $P$, Ljungberg $H$, et al. Early detection of cystic fibrosis lung disease: multiplebreath washout versus raised volume tests. Thorax 2007;62:341-7.

13. Gustafsson PM, Aurora P, Lindblad A. Evaluation of ventilation maldistribution as an early indicator of lung disease in children with cystic fibrosis. Eur Respir $\mathrm{J}$ 2003;22:972-9.

14. Matthys H, Keller R, Herzog H. Plethysmographic assessment of trapped air in man. Respiration 1970;27:447-61.

15. Gustafsson PM, Johansson HJ, Dahlback GO. Pneumotachographic nitrogen washout method for measurement of the volume of trapped gas in the lungs. Pediatr Pulmonol 1994;17:258-68.

16. Kraemer R, Blum A, Schibler A, et al. Ventilation inhomogeneities in relation to standard lung function in patients with cystic fibrosis. Am J Respir Crit Care Med 2005;171:371-8.

17. Kraemer R, Baldwin DN, Ammann RA, et al Progression of pulmonary hyperinflation and trapped gas associated with genetic and environmental factors in children with cystic fibrosis. Respir Res 2006;7:138.

18. Kraemer R, Zehnder M, Meister B. Intrapulmonary gas distribution in healthy children. Respir Physiol 1986;65:127-37

19. Habib RH, Lutchen KR. Moment analysis of a multibreath nitrogen washout based on an alveolar gas dilution number. Am Rev Respir Dis 1991;144:513-9.
20. Schibler A, Henning R. Positive end-expiratory pressure and ventilation inhomogeneity in mechanically ventilated children. Pediatr Crit Care Med 2002;3:124-8.

21. Schibler A, Hall GL, Businger F, et al. Measurement of lung volume and ventilation distribution with an ultrasonic flow meter in healthy infants. Eur Respir J 2002;20:912-8.

22. Gustafsson PM. Peripheral airway involvement in CF and asthma compared by inert gas washout. Pediatr Pulmonol 2007;42:168-76.

23. Aurora P, Gustafsson P, Bush A, et al. Multiple breath inert gas washout as a measure of ventilation distribution in children with cystic fibrosis. Thorax 2004,59:1068-73.

24. Van Muylem A, Baran D. Overall and peripheral inhomogeneity of ventilation in patients with stable cystic fibrosis. Pediatr Pulmonol 2000;30:3-9.

25. Verbanck S, Schuermans D, Van Muylem A, et al. Conductive and acinar lung-zone contributions to ventilation inhomogeneity in COPD. Am J Respir Crit Care Med 1998;157:1573-7.

26. Pillow JJ, Frerichs I, Stocks J. Lung function tests in neonates and infants with chronic lung disease: global and regional ventilation inhomogeneity. Pediatr Pulmonol 2006;41:105-21.

27. Buess C, Guggenbuhl W, Koller EA. A pulsed diagonal-beam ultrasonic airflow meter. J Appl Physiol 1989;67:2639.

28. Horsley AR, Gustafsson PM, Macleod KA, et al. Lung clearance index is a sensitive, repeatable and practical measure of airways disease in adults with cystic fibrosis. Thorax 2008;63:135-40.

29. Jonmarker C, Castor R, Drefeldt B, et al. An analyzer for in-line measurement of expiratory sulfur hexafluoride concentration. Anesthesiology 1985;63:84-8.

30. Larsson A, Linnarsson D, Jonmarker C, et al. Measurement of lung volume by sulfur hexafluoride washout during spontaneous and controlled ventilation: further development of a method. Anesthesiology 1987;67:543-50.

31. Dervos CT, Vassiliou P. Sulfur hexafluoride $\left(S_{6}\right)$ : global environmental effects and toxic byproduct formation. J Air Waste Manag Assoc 2000;50:137-41.

32. Lang CC, Karlin P, Haythe J, et al. Ease of noninvasive measurement of cardiac output coupled with peak $\mathrm{VO}_{2}$ determination at rest and during exercise in patients with heart failure. Am J Cardiol 2007:99:404-5

33. Beydon N, Davis SD, Lombardi E, et al. An official American Thoracic Society/European Respiratory Society statement: pulmonary function testing in preschool children. Am J Respir Crit Care Med 2007; 175:1304-45.

34. Edelman NH, Mittman C, Norris AH, et al. Effects of respiratory pattern on age differences in ventilation uniformity. J Appl Physiol 1968;24:49-53.

35. Frey U, Stocks J, Coates A, et al. Specifications for equipment used for infant pulmonary function testing. ERS/ATS Task Force on Standards for Infant Respiratory Function Testing. European Respiratory Society/American Thoracic Society. Eur Respir J 2000;16:731-40.

36. Brunner JX, Wolff G, Cumming G, et al. Accurate measurement of $\mathrm{N}_{2}$ volumes during $\mathrm{N}_{2}$ washout requires dynamic adjustment of delay time. J App/ Physiol 1985;59:1008-12.

37. Tang Y, Turner MJ, Baker AB. Effects of lung time constant, gas analyser delay and rise time on measurements of respiratory dead-space. Physiol Meas 2005;26:1103-14

38. Morris MG, Gustafsson P, Tepper R, et al. The bias flow nitrogen washout technique for measuring the functional residual capacity in infants. ERS/ATS Task Force on Standards for Infant Respiratory Function Testing. Eur Respir J 2001;17:529-36.

39. Verbanck S, Schuermans D, Paiva M, et al. Small airway function improvement after smoking cessation in smokers without airway obstruction. Am J Respir Crit Care Med 2006;174:853-7. 